\title{
New EBG Solutions for Mutual Coupling Reduction
}

\author{
Gonzalo Expósito-Domínguez*, José Manuel Fernández-González*, Pablo Padilla ${ }^{\dagger}$, Manuel Sierra-Castañer* \\ ${ }^{*}$ Radiation Group, Signals, Systems and Radiocommunications, Universidad Politécnica de Madrid, 28040, Spain. \\ Email: \{gexposito, jmfdez, mscastaner\}@gr.ssr.upm.es \\ ${ }^{\dagger}$ Department of Signal Theory, Telematics and Communications, Universidad de Granada, Granada 18071, Spain. \\ Email: pablopadilla@ugr.es
}

\begin{abstract}
Electromagnetic Band Gap (EBG) based on Frequency Selective Surfaces (FSS) [1] are one type of metamaterials [2] with electrical properties [3]. This EBG are used in mutual coupling reduction, back lobe radiation reduction, etc. In this work not only new shapes for the mushroom-type are presented, but also multilayered configurations were studied in order to reduce the patch size and the necessary number of elements.
\end{abstract}

\section{INTRODUCTION}

Low profile integrated antennas are requested to be small. In order to reduce the size of the antenna, high permittivity substrates are used. However with this type of substrates, surface wave propagation modes are enhanced and mutual coupling get worse. In steering antennas, separation between elements should not be higher than $0.6 \lambda$ in order to avoid grating lobes, this last condition deteriorates the mutual coupling because elements are closer and they have stronger interaction.

The most common mutual coupling reduction techniques are cavities, non uniform distribution feeding and Defected Ground Plane (DGP) but lately EBG structures are being used. To introduce several rows of EBG structures between two printed antenna has been found to increase the isolation [4]. However when low permittivity substrates are used in order to enhance the radiation efficiency, the size of the printed antenna increases, meanwhile the available space between printed antennas is not enough to introduce the necessary number of rows.

The main target of this work, is to find a new solution for reducing the size of the EBG (in this case mushroom type [1]). By applying multilayered structure, different shapes, and edge-location via the effective size is reduced and structures fit in between printed antennas for the minimum separation $(0.6 \lambda)$.

This paper gives an overview of the EBG metamaterials theory in section II. The section III is devoted to figure out solutions to reduce the size of EBG structures. In section IV EBG double layer edge-location via structure is introduced between two circular patches in order to reduce the mutual coupling. In section $\mathrm{V}$ prototypes were built and fully measured. Finally, in section VI the conclusions are drawn.

\section{EBG THEORY}

EBG technique appears as an application of truncated frequency selective surfaces (FSS) [5]. These structures consist of an array of metal protrusions on a flat metal sheet and can be visualized as mushrooms protruding from the surface (Fig. 1). When the period is small compared to the wavelength of interest, it is possible to analyze the material as an effective medium, with a surface impedance (metamaterial with electrical properties). These "mushrooms" present very high impedance for vertical an horizontal propagation modes at certain frequencies.

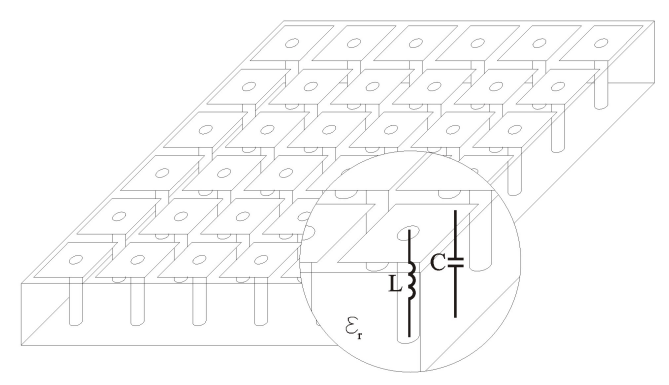

Fig. 1. High impedance surface and its model with parallel resonant LC circuit.

These structures can be analyzed as resonant LC circuits, in which the capacitance is provided by the proximity of the metal plates:

$$
C=\left[\frac{w \varepsilon_{0}\left(\varepsilon_{e f f}\right)}{\pi}\right] \cosh ^{-1}\left(\frac{2 w}{g_{0}}\right)
$$

and the inductance is related to the thickness of the structure, because its value is due to the length of the via:

$$
L=\mu_{0} t
$$

Therefore, the surface impedance is given by the following expression:

$$
Z_{s}=\frac{j \omega L}{1-\omega^{2} L C}
$$

and the resonance frequency of the circuit is given by:

$$
\omega_{0}=\frac{1}{\sqrt{L C}}
$$

Below resonance, the surface is inductive meanwhile above resonance, the surface is capacitive. Near $\omega_{0}$, the surface impedance is much higher than the impedance of free space [5], therefore no vertical or horizontal propagation modes are allowed. 


\section{Simulations}

Among others (filters or Artificial Magnetic Conductors AMC), EBG structures are used in order to reduce the mutual coupling between radiating elements similarly to [4], [6], [7] and [8]. To study this effect, a simulation scheme is prepared with a transmission line (TL) whose ground plane is replaced by an EBG plane (Fig. 2). This simulation is carried out for a fix permittivity $\varepsilon_{r}=2.17$ and different patch sizes $w$, number of periods $n$, gap sizes $g$ and substrate thickness $t$.

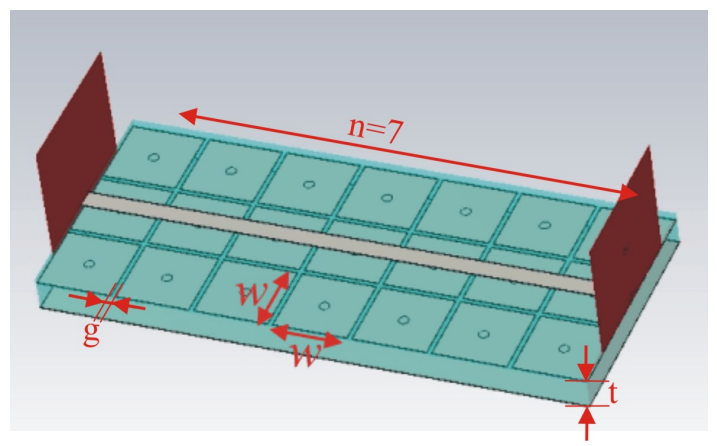

Fig. 2. Transmission line with EBG ground plane simulation scheme.

In Fig. 3 and Fig. 4 the transmission parameter $S_{21}$ for different patch sizes and number of periods is shown.

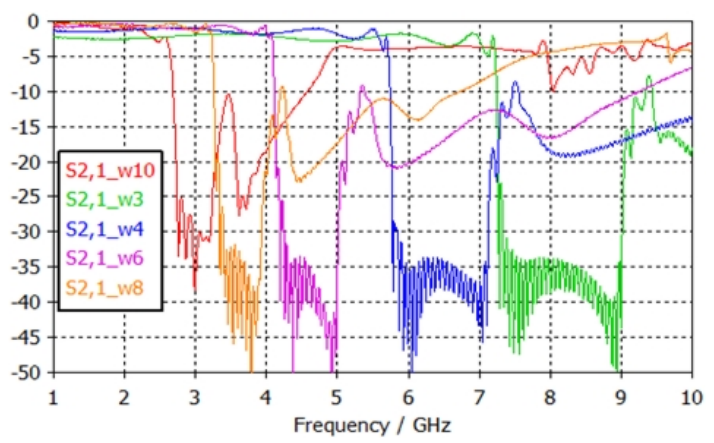

Fig. 3. $\mathrm{S}_{21}$ for a transmission line with EBG ground plane and different patch sizes $w$.

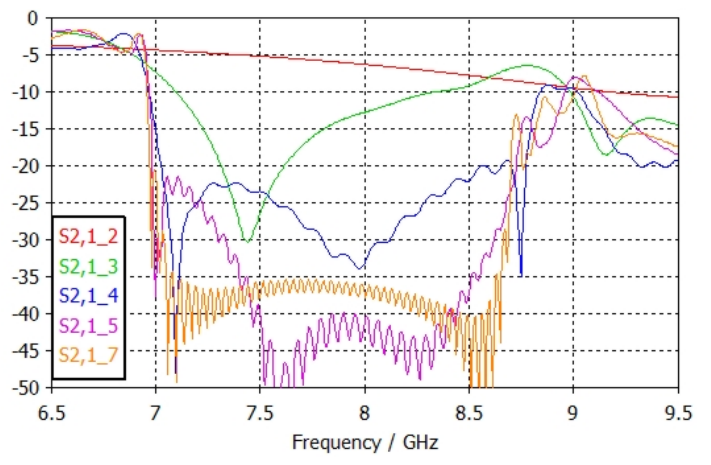

Fig. 4. $S_{21}$ for a transmission line with EBG ground plane and different number of mushrooms $n$.

The size of the patches and the necessary number of periods for mutual coupling reduction is higher than the space separation between radiating elements. In steering arrays antennas, separation is $0.5 \lambda$ for avoiding grating lobes. Therefore, new solution in order to reduce the size of the mushrooms has been found. In [9] by using edge-located vias the size of mushroom-type EBG is reduced by $20 \%$. In [10] a comparison of bandgaps between mushroom-type EBG surfaces and corrugated strip-type surfaces is shown. Finally in [11] two sizes of patches are mixed in order to get dual forbidden band.

In this work, new solution for mushroom size reduction is found. In order to maintain the working frequency ( $\mathrm{X}$ band) with the same substrate thickness and permittivity, the only parameter available is the capacitance $C$. In order to increase this parameter a multilayered structure is presented in Fig. 5(a) and Fig. 5(b).

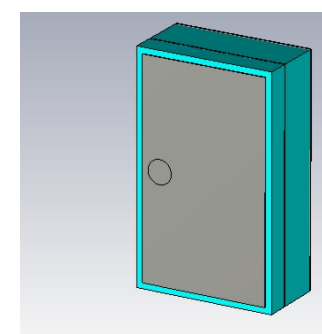

(a) Multilayered patch-shape

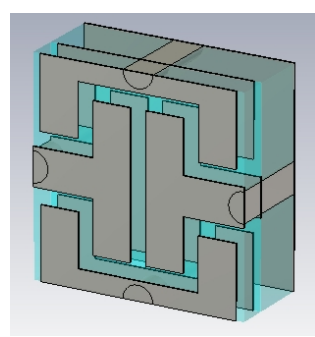

(b) Multilayered H-shape
Fig. 5. Multilayered mushroom-type solutions.

In Fig. 6 the transmission coefficient $\left(S_{21}\right)$ for different topologies and number of elements are presented. In spite of reducing the size of the patch, a reduction in the operation bandwidth can be seen in Fig. 6.

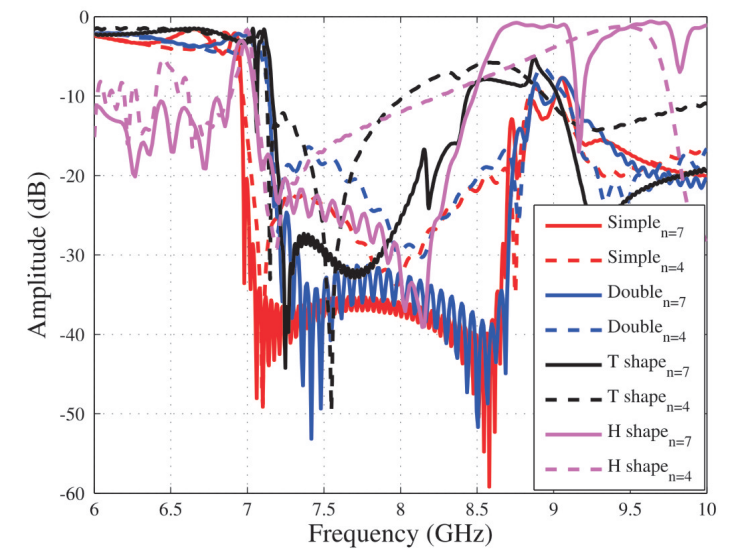

Fig. 6. Transmission coefficient for different mushroom topologies.

Finally a trade off has been found, $\mathrm{H}$ shape works well but its size is $4 \mathrm{~mm}$, meanwhile traditional shape has dimensions of $3 \mathrm{~mm}$. Double layer reduces $6 \%$ of the size $(2.8 \mathrm{~mm})$, and the combination of multilayered substrate and edge-located via gets a size reduction of $30 \%(2.1 \mathrm{~mm})$. This last structure has narrower operation bandwidth, nevertheless its small size will allow us to introduce it between printed antennas with low permittivity substrate. 


\section{Mutual COUPLing Reduction}

Four rows of EBG double layer edge-located via mushrooms where introduced between two round patches with double circular polarization (Fig. 12). The elements are fed by a branch line coupler $90^{\circ} 3 \mathrm{~dB}$ in order to get the double circular polarization. In Fig. 7(a) $|\mathrm{E}|$ field simulation for the 2 patches

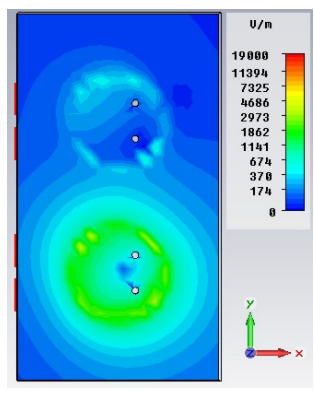

(a) Without EBG structure

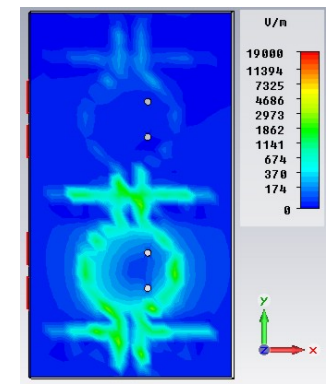

(b) With EBG structures
Fig. 7. $|\mathrm{E}|$ field simulation of 2 round patches with dual circular polarization.

is shown for Left Handed Circular Polarization (LHCP). In Fig. 7(b) it can be seen graphically, how $|\mathrm{E}|$ field value decays quicker when using EBG structures.

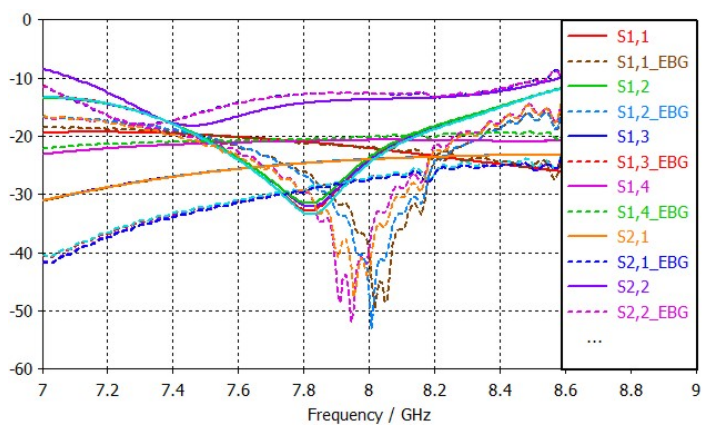

Fig. 8. Comparison of $\mathrm{S}$ parameters of 2 circular patches with dual circular polarization with, and without EBG structures in between.

Simulated $\mathrm{S}$ parameters also show a decrease of $-10 \mathrm{~dB}$ in coupling parameters, meanwhile reflection coefficient $\left(S_{i, i}\right)$ maintains its value.

\section{Measurements}

In order to validate the whole process, prototypes were built. First of all, test TLs with EBG ground plane were mounted. In Fig. 9 it can be seen 6 circuits. On the left hand side the two circuits are single layered, the two circuits in the middle are double layered, and the last two circuits on the right hand side combine double layer with edge-location via (T-shape). $\mathrm{H}$-shape is not built because dimensions did not fulfil at all the space requirements.

All the substrates used have permittivity of $\varepsilon_{r}=2.17$, however 4 different thickness were used. TLs impedance is 50 $\Omega$ and those TLs are printed in a $0.254 \mathrm{~mm}$ thick. Mushroom in single layer case are printed in $1.143 \mathrm{~mm}$ thick, meanwhile double layer case are printed in $0.762 \mathrm{~mm}$ thick (bottom layer) and $0.381 \mathrm{~mm}$ thick (upper layer). Therefore, total thickness maintains its value (Fig. 9).

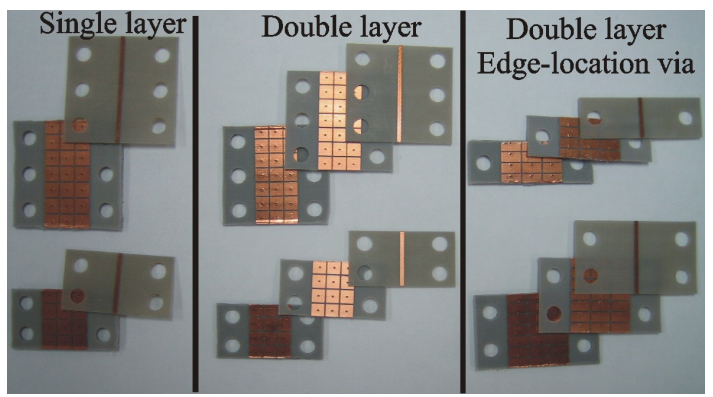

Fig. 9. Samples of single and multilayered EBG mushrooms with different shapes and number of elements.

The measured $\mathrm{S}$ parameters of the circuits are shown in Fig. 10. It can be notice, that measurements agree with simulations in Fig. 6. The larger the number of elements is, the higher the isolation is. Fig. 10 show also the reduction of bandwidth for T-shape.

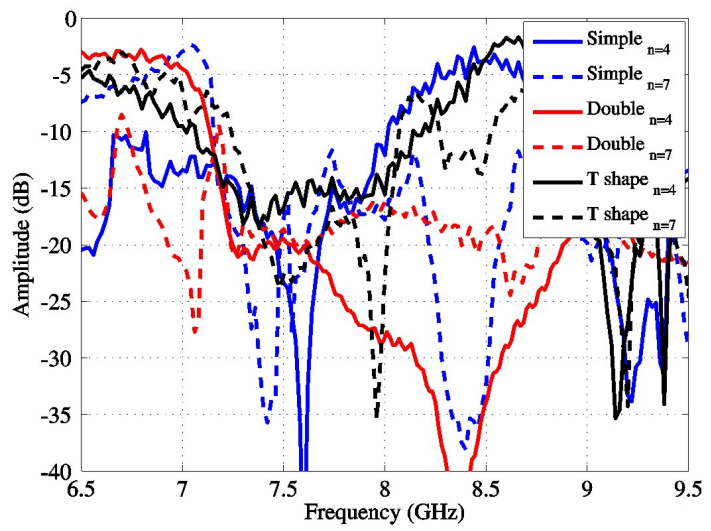

Fig. 10. Measurements of Transmission S parameters of different mushroom topologies.

In Fig. 11(a) and Fig. 11(b) the chosen topology is shown. With final dimensions of $2.1 \times 3.6 \mathrm{~mm}, 4$ elements, double layer structure and edge-location via, this configuration fulfils the requirements of available space between 2 printed antennas, bandwidth operation in X band $(7.25-8.5 \mathrm{GHz}, 15 \%)$ and $10 \mathrm{~dB}$ of isolation are obtained.

Secondly, 2 round patches fed by branch line coupler $90^{\circ}$ $3 \mathrm{~dB}$ with and without 4 rows of EBG T-shape mushroom are built (Fig. 12). This printed antennas are separated $0.6 \lambda$, are mounted with double stacked patch with permitivity of $\varepsilon_{r}=$ 2.17 and a foam layer between them of $4 \mathrm{~mm}$ in order to cover the whole bandwidth (15\%). The thickness of the substrates are: $1.143 \mathrm{~mm}$ (bottom patch) and $0.254 \mathrm{~mm}$ (upper patch).

Finally, S parameters measurements of $2 \times 1$ array antenna are presented in Fig. 13 when LHCP ports are excited and RHCP ports are loaded with $50 \Omega$ loads. There is a mutual coupling reduction between the two patches of $5 \mathrm{~dB}$ and an improvement of reflection coefficient $\mathrm{S}_{i, i}$ of $3 \mathrm{~dB}$ in most of the operation band. 


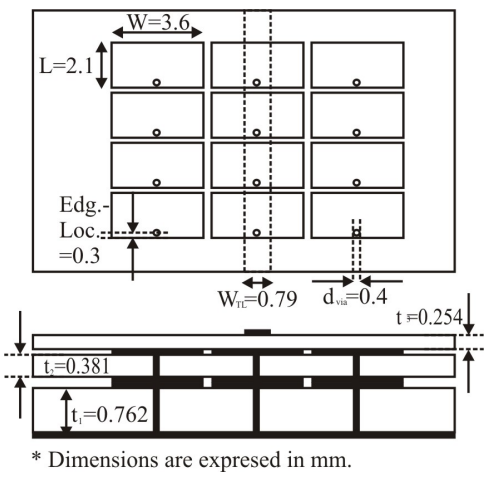

(a) Schematic

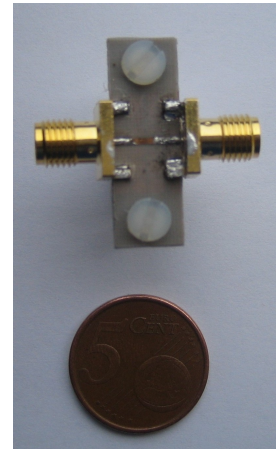

(b) Prototype
Fig. 11. Multilayered mushroom with rectangular shape, 4 elements and edge-located via (T-shape)

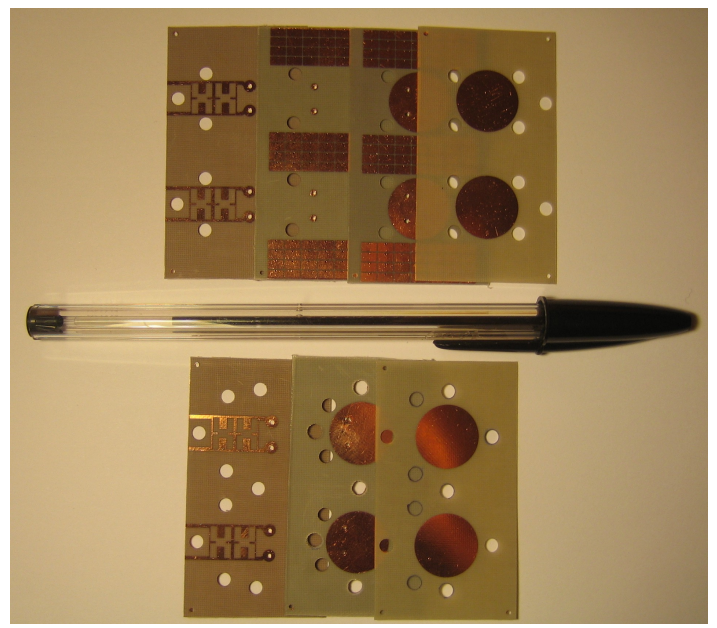

Fig. 12. 2x1 array of circular patches with and without EBG structures.

\section{CONCLUSIONS}

This paper presents and proposes a new mushroom shape in EBG structures. Full calculations and simulations are shown in order to choose a double layer, edge-located via mushroom. This solution gets $30 \%$ reduction of the size and works properly in wideband applications (15\% of bandwidth). Thanks to this reduction, EBG structures fit between 2 patches separated $0.6 \lambda$. Finally prototypes of different topologies and mutual coupling reduction scheme were built. Successful measurements of different topologies, and $5 \mathrm{~dB}$ of mutual coupling reduction and enhancement of reflection coefficient are obtained.

\section{ACKNOWLEDGMENT}

The simulations contained in this work have been carried out using CST Microwave Studio Suite 2011 under a cooperation agreement between Computer Simulation Technology (CST) and Universidad Politécnica de Madrid. We kindly thank the company NELTEC S.A. for giving samples of the substrates, in which the prototypes were built. This work is been supported by an UPM grant $\mathrm{CH} / 003 / 2011$, the project CG10-UPM/TIC5805, the CROCANTE project with reference TEC2008-

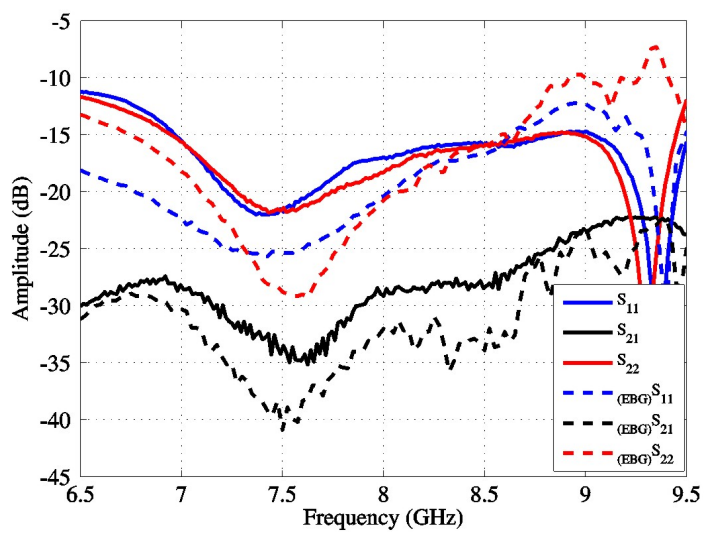

Fig. 13. Measurement comparison of $\mathrm{S}$ parameters $2 \times 1$ array of circular patches with and without EBG structures.

06736-C03-01, and the SICOMORO project with reference TEC2011-28789-C02-01.

\section{REFERENCES}

[1] D. Sievenpiper, L. Zhang, R. Broas, N. Alexopoulos, and E. Yablonovitch. High-impedance electromagnetic surfaces with a forbidden frequency band. IEEE Trans. On Microwave Theory and Techniques, 47:2059-2074, Nov. 1999.

[2] P.-S. Kildal. Definition of artificially soft and hard surfaces for electromagnetic waves. Electronics Letters, 24(3):168 -170, feb 1988.

[3] T. Itoh C. Caloz. Electromagnetic metamaterials: transmission line theory and microwave applications : the engineering approach. John Wiley and Sons, 2006.

[4] Fan Yang and Y. Rahmat-Samii. Microstrip antennas integrated with electromagnetic band-gap (ebg) structures: a low mutual coupling design for array applications. Antennas and Propagation, IEEE Transactions on, 51(10):2936-2946, Oct. 2003.

[5] F. Yang and Y. Rahmat-Samii. Electromagnetic Band Gap Structures in Antenna Engineering. The Cambridge RF and Microwave Engineering Series, 2008.

[6] E. Rajo-Iglesias, O. Quevedo-Teruel, and L. Inclán-Sánchez. Mutual coupling reduction in patch antenna arrays by using a planar ebg structure and a multilayer dielectric substrate. IEEE Trans. on Antennas and Propagation, 56(6):1648-1655, Jun. 2008.

[7] O. Quevedo-Teruel, L. Inclan-Sanchez, and E. Rajo-Iglesias. Soft surfaces for reducing mutual coupling between loaded pifa antennas. Antennas and Wireless Propagation Letters, IEEE, 9:91 -94, 2010.

[8] E. Rajo-Iglesias, O. Quevedo-Teruel, and L. Inclan-Sanchez. Planar soft surfaces and their application to mutual coupling reduction. Antennas and Propagation, IEEE Transactions on, 57(12):3852 -3859, dec. 2009.

[9] E.. Rajo-Iglesias, L.. Inclan-Sanchez, J.-L. Vazquez-Roy, and E. GarciaMuoz. Size reduction of mushroom-type ebg surfaces by using edgelocated vias. Microwave and Wireless Components Letters, IEEE, 17(9):670 -672, sept. 2007.

[10] E. Rajo-Iglesias, M. Caiazzo, L. Inclan-Sanchez, and P.-S. Kildal. Comparison of bandgaps of mushroom-type ebg surface and corrugated and strip-type soft surfaces. Microwaves, Antennas Propagation, IET, 1(1):184 -189, feb. 2007.

[11] E. Rajo-Iglesias, J.-L. Vazquez-Roy, O. Quevedo-Teruel, and L. InclanSanchez. Dual band planar soft surfaces. Microwaves, Antennas Propagation, IET, 3(5):742 -748, august 2009. 